\title{
Critical analysis of the empirical tests of local hidden-variable theories
}

\author{
Emilio Santos \\ Departamento de Física Moderna, Universidad de Cantabria, Santander, Spain \\ (Received 4 December 1991; revised manuscript received 18 May 1992)
}

\begin{abstract}
A local hidden-variable model is exhibited for the experiments by Aspect, Grangier, and Roger [Phys. Rev. Lett. 47, 460 (1981); 49, 91 (1982)] and Aspect, Dalibard, and Roger [Phys. Rev. Lett. 49, 1804 (1982)] measuring polarization correlation of optical-photon pairs. The model agrees with quantummechanical predictions for all measurable quantities even with ideal polarizers and detectors, and emphasizes the need of a high degree of directional correlation, besides the correlation of spin (or polarization or other quantities), in any test of locality. It is proved that homogeneous inequalities, involving only coincidence detection rates, cannot discriminate between quantum mechanics and local theories, which invalidates all previously used empirical tests. The role of supplementary assumptions, like the so-called no enhancement, for the derivation of Bell's inequalities is discussed. Finally it is conjectured that quantum mechanics might be compatible with local realism, if we assume that not all self-adjoint operators represent observables and not all density operators represent states.
\end{abstract}

PACS number(s): 03.65.Bz, 42.50.Wm, 42.50.Dv

\section{INTRODUCTION}

Several Bell inequalities [1] have been put to the test in the past few years and have been violated by experiments. (For reviews see Refs. [2-4]). As a consequence, it has been claimed in dozens of books and hundreds of articles that local-hidden-variable (LHV) theories have been empirically disproved or that local realism has been refuted. The purpose of this article is to analyze whether this repeated claim is correct.

The plan of the paper is as follows. In Secs. II and III we exhibit LHV models in agreement with quantum mechanics (QM) for all performed experimental tests of Bell inequalities. The existence of the models proves that such experiments are not suitable to discriminate between quantum mechanics and the whole family of LHV theories and, consequently, the LHV theories have not yet been disproved.

The model of Sec. II emphasizes the need of good directional correlation between the two signals (particles) used in the experiments. As the main class of experiments where violations of Bell inequalities have been reported consists of measuring polarization correlation of optical-photon pairs emitted in atomic transitions, I have analyzed - following a previous paper [5] - typical experiments in this class, such as those performed by Aspect and co-workers [6-8]. In Ref. [5], I exhibited a LHV model predicting almost the same as quantum mechanics for these experiments even in ideal situations, that is, using perfect polarization analyzers and 100\% efficient detectors. Here I propose an improvement of the model that now agrees exactly with quantum-mechanical predictions for all experiments measuring polarization correlation of optical-photon pairs.

The model of Sec. III emphasizes the need of using measuring devices sufficiently close to ideal. In that section we show that no experiment measuring only coincidence rates may disprove LHV theories. Both the need of directional correlation and the need of efficient measuring devices are rather well-known facts since the early studies of the problem, especially after the paper by Clauser and Horne [9]. However, these facts have been almost ignored in the recent literature.

The reason for the (wrong) claim that LHV theories (or local realism) have been empirically refuted is that people have considered restricted families of LHV theories that fulfill, in addition to Bell's conditions of locality [see below, Eqs. (2.6) and (2.7)], supplementary constraints like the Clauser and Horne "no-enhancement" assumption [9]. The use of the auxiliary assumptions has become a polemic matter, where personal opinions play a prominent role. Indeed, these assumptions have ranged from very plausible, almost compelling, to absolutely unjustified. In Sec. IV, I shall try to clarify the subject.

As stated above, the need of directional correlation between the particles involved in the experiments, in addition to polarization correlation, has not been sufficiently emphasized. This problem is revisited in Sec. $\mathrm{V}$ of the article.

The loophole for the refutation of LHV theories due to the use of nonideal devices, especially the low efficiency of optical-photon detectors, is widely recognized, but it has been generally considered a minor practical problem. In Sec. VI, I argue that this is not so by exhibiting a natural hidden-variable model of detection that predicts weaker correlations with high-efficiency detectors than with low efficiency ones. The model agrees with QM in the low-efficiency (linear) region but departs from it at high efficiencies and genuine (i.e., not involving supplementary assumptions) Bell inequalities are never violated.

Finally, in the last section, I discuss the consequences of this work. In particular, I conjecture that perhaps quantum mechanics is, after all, compatible with LHV theories, as defined by Bell.

In order to avoid misunderstandings, I should emphasize that the purpose of this article is not the ambi- 
tious one of proving that LHV theories able to interpret all experiments in the quantum domain actually exist, but the more humble aim of pointing out that the impossibility of such theories has not yet been shown. That is, we want to prove that the problem of LHV theories is still open, contrary to the received wisdom.

\section{LOCAL HIDDEN-VARIABLE MODEL FOR THE EXPERIMENTS MEASURING POLARIZATION CORRELATION OF OPTICAL-PHOTON PAIRS}

For the sake of clarity I shall consider the experiment by Aspect, Grangier, and Roger [7], which produced the maximum violation of a Bell inequality ever reported (by 40 standard deviations). The experiment involved twochannel polarization analyzers and the following coincidence rates were measured with polarization analyzers at (several pairs of) angles $a$ and $b: N_{++}(a, b)$ in the transmission channels of both analyzers; $N_{--}(a, b)$ in the reflection channels of both analyzers; and $N_{+-}(a, b)\left[N_{-+}(a, b)\right]$, in the transmission channel of the first (second) analyzer and the reflection channel of the second (first) analyzer.

Although not reported [7], it is also possible to measure single rates in these experiments. We may assume too that it is possible to measure, or estimate, the decay rate in the source. Then, the relevant quantities measurable in the experiment are the coincidence probabilities $p_{i j}(a, b)$ and single probabilities $p_{i}(a)$ and $p_{j}(b)$, where $i$ and $j$ stand for either + or - . They are obtained as ratios of the corresponding counting rates by the decay rate.

The quantum-mechanical predictions for these quantities are [2]

$$
\begin{aligned}
p_{++}(a, b) & =p_{--}(a, b) \\
& =\eta_{1} \eta_{2}(\Omega / 8 \pi)^{2} \alpha\left[\epsilon_{+}^{1} \epsilon_{+}^{2}+\epsilon_{-}^{1} \epsilon_{-}^{2} F \cos (2 a-2 b)\right], \\
p_{+-}(a, b) & =p_{-+}(a, b) \\
& =\eta_{1} \eta_{2}(\Omega / 8 \pi)^{2} \alpha\left[\epsilon_{+}^{1} \epsilon_{+}^{2}-\epsilon_{-}^{1} \epsilon_{-}^{2} F \cos (2 a-2 b)\right],
\end{aligned}
$$

$p_{+}(a)=p_{-}(a)=(\Omega / 8 \pi) \eta_{1} \epsilon_{+}^{1}$,

$p_{+}(b)=p_{-}(b)=(\Omega / 8 \pi) \eta_{2} \epsilon_{+}^{2}$,

where we have assumed identical lens systems in both sides of the apparatus, covering a solid angle $\Omega$ each. (This is the usual practice and, in any case, our analysis can be extended to experiments with two different angular apertures). The solid angle is related to the half angle $\varphi$ by

$$
\Omega=2 \pi(1-\cos \varphi) \text {. }
$$

The factors $F(\varphi)$ and $\alpha(\varphi)$ represent the depolarization and the angular correlation, respectively. The first factor [10] takes into account that the polarization correlation of a photon pair decreases when the angle between their wave vectors departs from $\pi$. The second factor is of order unity in the experiment considered here, due to the three-body character of the photon emission by the atom, and this fact is crucial for the existence of a LHV model. [In sharp contrast, $\alpha$ may be much larger in a two-body decay, the product $\alpha(\varphi) \Omega / 4 \pi$ being of order unity.]

The parameters $\epsilon_{+}^{j}, \epsilon_{-}^{j}\left(\eta_{j}\right)$ measure the efficiencies of the polarization analyzers (detectors). For ideal experiments all these parameters are equal to 1 . It is useful to define the new parameters

$$
\eta \equiv \frac{\eta_{1} \eta_{2} \epsilon_{+}^{1} \epsilon_{+}^{2} \alpha \Omega}{2 \pi\left(\eta_{1} \epsilon_{+}^{1}+\eta_{2} \epsilon_{+}^{2}\right)}, \quad \epsilon \equiv \frac{\epsilon_{-}^{1} \epsilon_{-}^{2}}{\epsilon_{+}^{1} \epsilon_{+}^{2}} F,
$$

so that every possible experiment can be represented by a point in a plot of $\epsilon$ against $\eta$, as in Fig. 1 .

The explicit forms of the functions $\alpha(\varphi)$ and $F(\varphi)$ depend on the multipole nature of the photon emission process. In the experiment discussed here as well as in most polarization correlation experiments, both the initial and the final atomic states have zero angular momentum and the decay proceeds via an intermediate state of angular momentum 1. In this case, the functions $\alpha(\varphi)$ and $F(\varphi)$ are

$$
\begin{aligned}
& \alpha(\varphi)=1+\frac{1}{8} \cos ^{2} \varphi(1+\cos \varphi)^{2} \\
& F(\varphi) \cong 1-\frac{2}{3}(1-\cos \varphi)^{2} .
\end{aligned}
$$

The expression of $F(\varphi)$ is cumbersome [10], but the approximation here reported is very good in the most relevant range $0 \leq \varphi \leq \pi / 6$. The quantum predictions (2.1) and (2.4) for the experiments under discussion never violate a genuine Bell inequality like

$p\left(a^{\prime}\right)+p(b) \geq p(a, b)+p\left(a, b^{\prime}\right)+p\left(a^{\prime}, b^{\prime}\right)-p\left(a, b^{\prime}\right)$

(see Fig. 1). This fact was already pointed out by Clauser and Horne [9], but as the Bell inequalities are not sufficient conditions for the existence of LHV models, it

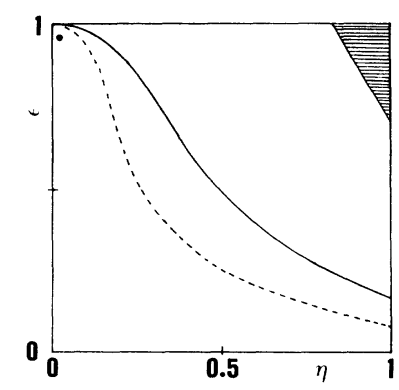

FIG. 1. Polarization correlation vs angular correlation of photon pairs. In experiments using photons from atomic emissions, the parameters $\epsilon$ and $\eta$ are related to the functions $\Omega(\varphi)$, $\alpha(\varphi)$, and $F(\varphi)$ and the efficiencies of polarization analyzers and detectors by Eqs. (2.3). The solid line corresponds to atomic emissions of the type $J=0 \rightarrow 1 \rightarrow 0$ [with $\alpha$ and $F$ given by Eqs. (2.4)] and the dashed line corresponds to processes $J=1 \rightarrow 1 \rightarrow 0$, both with ideal polarizers and detectors. Real experiments lie at the left of the corresponding curves. The dashed region at the upper right corner is forbidden by the Bell inequality (2.5). The small circle at the left upper corner represents the experiment by Aspect, Grangier, and Roger [7]. 
has remained unknown whether these models exist. Here I prove the existence by constructing an explicit one. According to Bell, a LHV model consists of a set of hidden variables $\lambda$ and some functions $\rho(\lambda), P_{j}(\lambda, a), P_{j}(\lambda, b)$, with $j=+$ or - , fulfilling the conditions

$$
\rho(\lambda) \geq 0, \quad \int \rho(\lambda) d \lambda=1, \quad 0 \leq P_{i}(\lambda, a), P_{j}(\lambda, b) \leq 1 .
$$

The measurable probabilities should be obtained as follows:

$$
\begin{aligned}
& p_{i j}(a, b)=\int P_{i}(\lambda, a) P_{j}(\lambda, b) \rho(\lambda) d \lambda, \\
& p_{i}(a)=\int P_{i}(\lambda, a) \rho(\lambda) d \lambda, \\
& p_{j}(b)=\int P_{j}(\lambda, b) \rho(\lambda) d \lambda .
\end{aligned}
$$

I shall exhibit a LHV model in perfect agreement with the quantum-mechanical predictions $(2.1)$ for ideal experiments, i.e., with $\epsilon_{+}^{j}=\epsilon_{-}^{j}=\eta_{j}=1$. We take $\lambda$ to be the set of four variables $\left\{\mu_{1}, \mu_{2}, v_{1}, v_{2}\right\}$ with ranges

$$
\mu_{1}, \mu_{2} \in[0, \pi] ; \quad v_{1}, v_{2} \in[0,1]
$$

and the functions mentioned above are given by

$$
\begin{aligned}
& \rho=\pi^{-2}\left[1+\cos \left(2 \mu_{1}-2 \mu_{2}\right)\right] \delta\left(v_{1}-v_{2}\right), \\
& P_{+}(\lambda, a)= \begin{cases}\beta & \text { if }\left|\mu_{j}-a\right| \leq \gamma(\bmod \pi) \text { and } v_{j} \leq \xi \\
0 & \text { otherwise }\end{cases}
\end{aligned}
$$

$$
P_{-}(\lambda, a)=P_{+}(\lambda, a+\pi / 2),
$$

and similar expressions for $P_{+}(\lambda, b)$ and $P_{-}(\lambda, b)$.

It is a trivial matter to obtain the predictions of the model in terms of the parameters $\beta, \gamma, \xi$ and the result is

$$
\begin{aligned}
& p_{++}(a, b)=p_{--}(a, b) \\
&=\pi^{-2} \beta^{2} \xi^{2}\left[4 \gamma^{2}+\sin ^{2}(2 \gamma) \cos (2 a-2 b)\right], \\
& p_{+-}(a, b)=p_{-+}(a, b)=p_{++}(a, b+\pi / 2) \\
& p_{+}(a)=p_{-}(a)=p_{+}(b)=p_{-}(b)=2 \gamma \beta \xi / \pi
\end{aligned}
$$

These probabilities agree exactly with the quantummechanical predictions, Eqs. (2.1), provided the parameters fulfill the conditions

$$
\xi=\alpha^{-1}, \quad \sin (2 \gamma)=2 \gamma \sqrt{F}, \quad \gamma \beta=\Omega \alpha / 16 .
$$

In the experiment, Eqs. (2.4) and (2.11) lead to

$$
\gamma \cong \sqrt{2} \Omega / 4 \pi, \quad \beta \cong \sqrt{2} \alpha \pi / 8 \lesssim 0.83, \quad \Omega<4 \pi,
$$

which shows that the conditions (2.6), imposed by local realism, are indeed fulfilled. In particular, $\beta$ is smaller than 1 , as it should be. In fact, for lens systems covering a small solid angle, $\beta$ remains close to 0.8 , while it decreases for large solid angles. On the other hand, $\gamma$ remains always smaller than $\pi / 2$. These features are also true if we use the exact form of $F(\varphi)$, as given by Clauser et al. [10], instead of the approximation (2.4).

The LHV model is able to reproduce also the quantum-mechanical predictions for other quantities not measured in the experiment, such as coincidence probabilities in the two channels of the same analyzer, predicted to be zero by quantum mechanics, as well as triple or quadruple coincidence probabilities, also predicted to be zero. (Actually, the model predicts a nonzero value for the last coincidence probabilities at very large solid angles, well above those actually used in the experiments, but very likely a LHV model could be found reproducing the quantum results also in this case.)

Similar LHV models can be constructed for all other experiments measuring polarization correlation of optical-photon pairs, which have used one-channel analyzers. In these experiments [2-4], besides the coincidence rates $N(a, b)$ with the two polarizers in place, the coincidence rate was measured with one polarizer removed, $N(a, \infty)$ or $N(\infty, b)$, or with both removed, $N(\infty, \infty)$. An explicit model for these experiments has been proposed recently [11], which allows the calculation of the corresponding probabilities $p(a, b), p(a, \infty)$, $p(\infty, b)$, and $p(\infty, \infty)$. The model contains, besides the functions $P(\lambda, a)$ and $P(\lambda, b)$, given by Eqs. (2.9) (the new functions $P$ correspond to the old $P_{++}$), the additional functions

$$
P_{j}(\lambda, \infty)=\left\{\begin{array}{l}
\alpha(\varphi) \Omega / 4 \pi \text { if } v_{j} \leq \alpha^{-1}, j=1 \text { or } 2 \\
0 \text { otherwise }
\end{array}\right.
$$

which allow the calculation of the coincidence probabilities with zero, one, or both polarization analyzers in place. It is a simple matter to check that this model reproduces exactly the quantum-mechanical predictions for these experiments.

Some of the experiments [2] have used atomic decays of type $J=1 \rightarrow 1 \rightarrow 0$, instead of $J=0 \rightarrow 1 \rightarrow 0$ used by Aspect, Grangier, and Roger [7], which modifies the dependence on $\varphi$ of the factors $\alpha$ and $F$ [no longer given by Eqs. (2.4), see Fig. 1]. We have not worked a LHV model for these experiments, but it is surely possible. Indeed, as shown in Fig. 1, these experiments lie still farther from the region forbidden by the Bell inequality (2.5).

\section{HOMOGENEOUS AND INHOMOGENEOUS BELL INEQUALITIES}

In this section we exhibit a general LHV model that agrees with quantum predictions for all performed experimental tests of Bell inequalities. This model is possible because all inequalities actually tested have been homogeneous, comparing several coincidence probabilities among themselves, while genuine Bell inequalities should be inhomogeneous, that is, they should compare coincidence probabilities with either single probabilities or numbers. For instance, the Bell inequality derived by Clauser et al. [10] is inhomogeneous,

$$
\begin{aligned}
& |E(a, b)+E(c, b)+E(a, d)-E(c, d)| \leq 2, \\
& E(a, b)=p_{++}(a, b)+p_{--}(a, b)-p_{+-}(a, b) \\
& \quad-p_{-+}(a, b),
\end{aligned}
$$


where $p_{++}$, etc. have the same meaning as in Sec. II. Aspect, Grangier, and Roger [7] quoted this inequality but the one they actually tested was the homogeneous inequality

$$
\begin{aligned}
& \left|E^{\prime}(a, b)+E^{\prime}(c, b)+E^{\prime}(a, d)-E^{\prime}(c, d)\right| \leq 2 \\
& E^{\prime}=\left(N_{++}+N_{--}-N_{+-}-N_{-+}\right)\left(N_{++}+N_{--}\right. \\
& \left.+N_{+-}+N_{-+}\right)^{-1}
\end{aligned}
$$

$N_{++}$, etc. being coincidence counting rates. The need of inhomogeneous inequalities can be seen from the following argument.

We define an Einstein-Podolsky-Rosen [3] (EPR) experiment as the preparation of a state of two particles and the measurement (at spacelike separation) of a finite set of pairs of observables, one member of the pair belonging to the set $\left\{a_{j}, j=1, \ldots m\right\}$ of observables of the first particle and another member to the set $\left\{b_{k}, k=1, \ldots n\right\}$ of the second particle, all observables having range $\{0,1\}$. The observables $a_{j}$ are not pairwise compatible, in general, and the same is true for the $b_{k}$, so that different pairs should be measured in different runs of the experiment (with identically prepared two-particle systems). Thus, as a result of one run we should obtain the following three independent probabilities: $p\left(a_{j} b_{k}\right)$, that both observables take the value 1 and $p\left(a_{j}\right)\left[p\left(b_{k}\right)\right]$, that the first [second] observable takes the value 1 with any value [ 1 or 0 ] for the second [first] observable. We also demand an operational meaning for the observables involved by requiring that each one corresponds to an actual apparatus that records a count whenever the observable takes the value 1 for a given pair of correlated particles produced in the source.

We argue that all performed experimental tests of Bell inequalities, at least those in the optical domain, have tested homogeneous inequalities of the form

$$
\sum_{j, k} c_{j k} p\left(a_{j} b_{k}\right) \geq 0,
$$

where $c_{j k}$ are real numbers. That is, these inequalities do not include the single probabilities $p\left(a_{j}\right)$ or $p\left(b_{k}\right)$. (As stressed above, genuine Bell inequalities are always inhomogeneous.)

The probabilities $p\left(a_{j} b_{k}\right)$ should be measured as ratios between coincidence counting rates and the production rate in the source. However, the homogeneous nature of (3.3) allows tests of that inequality measuring only coincidence counting rates, which involve far less uncertainties than measuring single rates. Also, the inequality is insensitive to many scale factors, such as detector efficiencies, angular apertures, etc. All these features are extremely convenient from the practical point of view, and this fact explains why all performed experiments have tested homogeneous inequalities. In contrast, testing inhomogeneous inequalities demands devices close to ideal.

We define a scaled-LHV model of an EPR experiment as consisting of one (or several) real variables $\lambda$ and several real functions $p(\lambda), P\left(\lambda, a_{j}\right)$, and $P\left(\lambda, b_{k}\right)$, fulfilling the conditions [compare with (2.6)]

$$
\int \rho(\lambda) d \lambda=1, \quad \rho(\lambda) \geq 0, \quad P\left(\lambda, a_{j}\right) \geq 0, \quad P\left(\lambda, b_{k}\right) \geq 0,
$$

and such that the model predictions for the functions $p\left(a_{j} b_{k}\right)$ are obtained as

$$
p\left(a_{j} b_{k}\right)=\int \rho(\lambda) P\left(\lambda, a_{j}\right) P\left(\lambda, b_{k}\right) d \lambda .
$$

Instead of (3.4) we may write the conditions in the form

$$
\rho^{\prime}(\lambda) \geq 0, \quad 0 \leq P^{\prime}\left(\lambda, a_{j}\right) \leq 1, \quad 0 \leq P^{\prime}\left(\lambda, b_{k}\right) \leq 1,
$$

with $\rho^{\prime}, P^{\prime}\left(\lambda, a_{j}\right)$, and $P^{\prime}\left(\lambda, b_{k}\right)$, giving the same prediction Eq. (3.5). In fact, for a fixed $p\left(a_{j} b_{k}\right)$, it is always possible to find $\rho^{\prime}, P^{\prime}\left(\lambda, a_{j}\right)$, and $P^{\prime}\left(\lambda, b_{k}\right)$, fulfilling (3.5) and (3.6) given $\rho, P\left(\lambda, a_{j}\right)$, and $P\left(\lambda, b_{k}\right)$, fulfilling (3.4) and (3.5), and vice versa.

Following Bell, a LHV model is defined as a scaledLHV model fulfilling, besides (3.4) [or (3.6)] and (3.5), the conditions

$$
\int \rho(\lambda) d \lambda=1, \quad P\left(\lambda, a_{j}\right) \leq 1, \quad P\left(\lambda, b_{k}\right) \leq 1 .
$$

We can prove the following results.

Theorem 1. Every EPR experiment admits a scaledLHV model.

Proof. We associate with the pair of observables $\left\{a_{j}, b_{k}\right\}$ the real number

$$
r_{j k}=[n(j-1)+k] /[m n+1]
$$

and define the functions involved in (3.4) by

$$
\begin{aligned}
& \rho(\lambda)= \begin{cases}1 & \text { if } \lambda \in[0,1] \\
0 & \text { otherwise },\end{cases} \\
& P\left(\lambda, a_{j}\right)=\sum_{l} q\left(a_{j} b_{l}\right) \delta\left(\lambda-r_{j l}\right), \quad P\left(\lambda, b_{k}\right)=\sum_{i} \delta\left(\lambda-r_{i k}\right),
\end{aligned}
$$

where $q\left(a_{j} b_{k}\right)$ is the quantum prediction for $p\left(a_{j} b_{k}\right)$, and $\delta(x)$ is the function

$$
\delta(x)=\left\{\begin{array}{l}
(2 \epsilon)^{-1 / 2} \text { if }-\epsilon \leq x \leq \epsilon \\
0 \text { otherwise, with } \epsilon \leq[2 m n+2]^{-1} .
\end{array}\right.
$$

It is easy to see that this scaled-LHV model agrees exactly with quantum mechanics for the considered EPR experiment, which ends the proof.

Theorem 2. If the inequality (3.3) is violated by quantum mechanics, it is also violated by a LHV model.

Proof. We consider the LHV model with $\rho(\lambda)$ given by Eq. (3.9) and the functions $P\left(\lambda, a_{j}\right)$ by

$$
\begin{aligned}
& P\left(\lambda, a_{j}\right)=\sqrt{(2 \epsilon)} \sum_{l} q\left(a_{j} b_{l}\right) \delta\left(\lambda-r_{j l}\right), \\
& P\left(\lambda, b_{k}\right)=\sqrt{(2 \epsilon)} \sum_{i} \delta\left(\lambda-r_{i k}\right) .
\end{aligned}
$$

This is a LHV model that violates (3.3) if QM does.

The reader may wonder at the fact that, if we include the identity observables $I$ (taking always the value 1 ) then

$$
p\left(a_{j} I\right)=p\left(a_{j}\right), \quad p\left(I b_{k}\right)=p\left(b_{k}\right) .
$$


In this case the (inhomogeneous) Bell inequalities also have the form (3.3), which leads to a contradiction between Bells' theorem and ours. Actually, if we were including the identity observable $I$, we should add to the conditions (3.4) [or (3.6)] and (3.7) the following:

$$
P(\lambda, I)=1,
$$

and our theorems could not be proved. Therefore we have explicitly excluded the identity from (3.3) by our required operational definition of the observables. Thus our theorems hold true for all inequalities that can be tested empirically by measuring only coincidence counting rates.

We conclude that it is not possible to discriminate between quantum mechanics and LHV models by means of experiments designed to test only homogeneous inequalities like (3.3), contrary to frequent claims in the literature. Consequently, none of these experiments can refute local-hidden-variable theories.

From theorem 1 we see that the conditions (3.7) are essential for the derivation of genuine Bell inequalities. From theorem 2 we see that homogeneous inequalities are insensitive to the scale and do not properly take into account the constraints (3.7).

\section{INEQUALITIES INVOLVING SUPPLEMENTARY ASSUMPTIONS}

Many readers may be surprised by the contradiction between the claimed violation of Bell's inequalities by the experiments measuring polarization correlation of optical-photon pairs, and the existence of LHV models in perfect agreement with QM for the measured quantities, as those exhibited in Secs. II and III. The existence of the models clearly shows that the commented experiments cannot discriminate between quantum mechanics and LHV theories. What is really surprising is that this fact was already pointed out by Clauser and Horne [9] in 1974 and, nevertheless, many people have maintained the view that local realism has been empirically refuted. Of course, the existence of a "low-efficiency" loophole [2-4] that could only be blocked with detectors having efficiency above $82 \%$ [12] has been widely recognized. For instance, at the end of the report of the experiment by Aspect, Grangier, and Roger [7], commented on in Sec. II, it is stated that "Only two loopholes remain open. The first one, exploiting the low efficiency of detectors... . The second one, exploiting the static character of all previous experiments. ..." (The second loophole was blocked in a subsequent experiment [8].) This statement is clearly wrong, because the LHV model exhibited in Sec. II relies upon none of these loopholes. In particular, the model agrees with quantum predictions even for $100 \%$ efficient detectors. The emphasis on the lowefficiency loophole has obscured the most relevant fact, namely that experiments of the type above described are not suitable tests of the whole family of LHV theories even with ideal apparatuses.

The confusion rests upon the unclear physical meaning of the auxiliary assumptions involved in the interpretation of the experiments. It is true that any real experi- ment involves a lot of assumptions about the functioning of the measuring devices, the origin of the noise, and so on. But it is obvious that this fact does not justify the use of whatever assumption one wants, and that every assumption used for the interpretation of the experiments should be carefully scrutinized. In any case, I agree that the role of auxiliary assumptions about hidden variables is not at all trivial, as shown by the historical development of the subject. In fact, as is well known, von Neumann [13] very early proved a theorem of impossibility of hidden variables. However, 20 years later Bohm proved by means of a counterexample that the theorem involved assumptions that were too restrictive. Additional impossibility theorems were proved in the decade that followed (by Gleason, Jauch, etc.) involving weaker assumptions than von Neumann's. These theorems were also criticized for using too-restrictive assumptions, this time by Bell [14], who proved that general (contextual) hiddenvariable theories are always possible. In view of this result Bell himself [1] changed his interest to the family of local hidden variables. The mistake made in the past with respect to the whole family of hidden variables has been repeated in recent times with the local ones. It has been claimed many times that local theories have been empirically refuted (modulo the low-efficiency loophole). My aim is to show that, again, these impossibility proofs involve unnecessarily restrictive assumptions.

When the search for experimental tests of the Bell inequalities began, after Bell's work [1], it was soon realized that only extremely difficult experiments might actually show violations of the Bell inequalities. Indeed, after several years of work no experiment was found able to discriminate between quantum mechanics and the whole family of LHV theories. Then the idea was put forward [10] of using supplementary assumptions about the behavior of the hidden variables in order to derive inequalities able to contradict quantum mechanics in real experiments. The auxiliary assumptions have been discussed extensively [3], but as some confusion still exists, a clarification is worthwhile in the light of the models of Secs. II and III.

Several related supplementary assumptions have been proposed by different authors. All the LHV models described in Secs. II and III violate, like quantum mechanics, some inequality derived from one of these assumptions, plus Bell conditions for LHV theories, Eqs. (2.6) and (2.7). As these models do not violate Bell's condition, they should violate every one of the supplementary assumptions. In the following we show explicitly the violation of the four more popular auxiliary assumptions.

(i) If a pair of photons emerges from the polarizers, the probability of their joint detection is independent of the polarizer orientations $a$ and $b$ (Clauser et al. [10]). With the notation used in Sec. II, this can be written

$$
P_{1}(\lambda, a) P_{2}(\lambda, b)=M(\lambda)
$$

Violation of (i): In the models $P(\lambda, a)$ is given either by Eq. (3.12) or by $P_{+}(\lambda, a)$ or $P_{-}(\lambda, a)$ of Eq. (2.9) [and similarly for $P(\lambda, b)$, substituting $b$ for $a$ ]. It is clear that the product (4.1) depends on $a$ and $b$, although its aver- 
age over $\lambda$, which is the measurable quantity, does not depend.

(ii) For every pair of emissions (i.e., for each value of $\lambda$ ), the probability of a count with a polarizer in place is less than or equal to the corresponding probability with the polarizer removed (no-enhancement assumption of Clauser and Horne [9]). That is,

$$
P_{j}(\lambda, a) \leq P_{j}(\lambda, \infty) \text {. }
$$

Violation of (ii): Any of the functions $P_{j}(\lambda, a)$ of Eq. (2.9) surpasses the value of $P_{j}(\lambda, \infty)$ given by Eq. (2.13) for some values of $\lambda$ and $a$, which means that "enhancement" in detection exists in some cases. Again, the average over $\lambda$ fulfills no enhancement. The violation is also obvious in Eqs. (3.12) if we substitute $a(\infty)$ for $a_{1}\left(a_{2}\right)$.

(iii) For every photon in the state $\lambda$ the sum of detection probabilities in the ordinary and in the extraordinary beams emerging from a two-way polarizer does not depend on the polarizer's orientation. (Garuccio and Rapisarda [15].) That is,

$$
P_{+}(\lambda, a)+P_{-}(\lambda, a)=M(\lambda) .
$$

An assumption similar to this one has been extended by Grangier, Potasek, and Yurke [16] to experiments involving correlated phases, instead of correlated polarizations, and it has become very popular [17].

Violation of (iii): The sum of $P_{+}$and $P_{-}$given by Eq. (2.9) is sometimes $\beta$ and sometimes 0 depending on the polarizer orientation $a$. The same happens in Eqs. (3.12) writing $P_{+}(\lambda, a) \quad\left[P_{-}(\lambda, a)\right]$ instead of $P\left(\lambda, a_{1}\right)$ $\left[P\left(\lambda, a_{2}\right)\right]$.

(iv) The ensemble of actually detected pairs is a faithful sample of all emitted pairs (Faithful sampling assumption of Aspect, Grangier, and Roger [7]). This assumption is somewhat ambiguous, because the meaning of "faithful" is not sharp. It seems that we should interpret it as making reference to the polarization correlation of all emitted photon pairs, but then it is obviously wrong. In fact, the photon pairs that are actually collected by the apertures move in opposite (or almost opposite) directions and they are predicted by quantum mechanics to have a strong polarization correlation [this is the reason for the value close to 1 of the function $F$ defined in Eq. (2.4)]. In contrast, the average polarization correlation of all photon pairs is much weaker due to the poor angular correlation and it gives $F \approx \frac{1}{3}$, as shown in Fig. 1 (right end of the continuous curve). Actually, Aspect, Grangier, and Roger [7] did not use assumption (iv) as above stated, but a form of "faithful sample" assumption restricted to the subensemble of photon pairs that have passed the apertures, as should be clear by their statement that the ratio $E / E^{\prime}$ [this ratio is the sum of four counting rates divided by the decay rate, see our Eqs. (3.1) and (3.2)] is independent of $a$ and $b$. This statement is close to the assumption (iii) above.

Violation of (iv): The assumption would imply that the probabilities $P_{j}^{\prime}(\lambda, a)$ and $P_{j}^{\prime}(\lambda, a)$ for the "passed subensemble" would be given by the ratios $P_{j}(\lambda, a) /$ $P_{j}(\lambda, \infty)(j=+$ or -$)$. But these ratios are sometimes greater than 1, as explained in relation to the violation of (i). Therefore the passed subensemble cannot exist and "faithful sampling" is meaningless in relation with it.

A common feature of these assumptions is that every one of them follows from:

(A) A linear extrapolation rule, which I state as follows: If the probability of a single (coincidence) detection event is $P$ when measured with one detector (two detectors) having photon efficiency $\eta$, then the probability measured with ideal detectors would be $P / \eta\left(P / \eta^{2}\right)$. I propose this rule as a precise substitute for the faithful sampling assumption.

(B) A mechanistic picture of the photon, which takes it to be a (billiard-ball-like) particle that, starting in the source, either passes undivided through, or is stopped at, the apertures, lenses, filters, and polarizers. When this assumption is applied to the experiments under discussion, it allows defining the "ensemble of those photon pairs such that each member of the pair enters the corresponding aperture." We shall call this the hypothesis of the passed subensemble.

The combination of (A) and (B) implies (i) to (iv), as we prove in the following. Assumption (i) follows from the fact that, given the experimental set-up, all photon pairs that have both passed the polarizers [hypothesis (B) implies that this "passage" has a meaning], will arrive at the detectors. With $100 \%$ detector efficiency, the joint detection probability of these photons would be, therefore, $P=1$. With detectors of efficiency $\eta$, the corresponding probability would be $P=\eta^{2}$, i.e., a constant independent of polarizer orientations. Similarly for assumption (ii). With ideal detectors, the detection probability of a photon entering the apertures will be 1 if the polarizer has been removed. With the polarizer in place the probability cannot be greater than 1 and this means no enhancement. Assumption (iii) clearly derives from (A) if the detectors are $100 \%$ efficient, whence it follows also for $\eta \neq 1$ according to (B). The faithful sampling assumption (iv) seems to be just equivalent to (A) as stated by Aspect, Grangier, and Roger [7], but we have seen that actually it involves also the idea of passed subensemble contained in (B).

We see that, at least, two independent loopholes for the refutation of LHV theories remain open. The first one, due to the low efficiency of optical-photon detectors, makes the faithful sampling hypothesis (A) necessary. The other one, due to the poor angular correlation makes the passed subensemble hypothesis (B) necessary. We conclude that LHV theories remain possible, after the performed experiments, provided we reject either the mechanistic or naive corpuscular picture of the photon, assumption (B), the extrapolation rule (A), or both. We now comment on the (im)plausibility of these assumptions.

The mechanistic view of the photon is so strongly attached to the current quantum paradigm that most theoretical physicists do not even question its validity (amongst the exceptions, there are many workers in quantum optics) in spite of strong arguments against it in standard quantum theory [18]. Consequently, the auxiliary assumptions (i) to (iv) have been considered equivalent to the faithful sampling hypothesis (A) and 
therefore are taken as very plausible. This has lead to the erroneous belief that the low-efficiency loophole was the only one remaining after the celebrated experiment by Aspect, Dalibard, and Roger [8]. As emphasized before, this confusion should not exist because the distinction between the low efficiency and the "poor angular correlation" loopholes was clearly stated by Clauser and Horne [9]. We think that also the faithful sampling assumption is very likely false in dealing with photons, as we shall discuss in Sec. VI.

All these criticisms do not imply that experimental tests of inequalities involving supplementary assumptions have been useless. On the contrary, they have been highly valuable, but their real value has been to provide constraints on the possible LHV theories. For instance, the performed experiments on polarization correlation of photon pairs provide a lot of information about possible LHV theories of quantum optics. This has allowed us to develop a local realistic alternative (or reinterpretation) of quantum optics [19], which explains qualitatively all phenomena involving the so-called nonclassical behavior of light. This theory, however, is only at a preliminary stage and it is not yet possible to know whether it will agree completely with quantum optics or might be distinguished empirically from it.

\section{ROLE OF LOCALITY IN THE BELL INEQUALITIES}

Genuine tests of LHV theories should always involve experiments of the type envisaged by Einstein, Podolsky, and Rosen [3]. In EPR experiments, we should measure two dichotomic observables (for instance two spin projections) in two separated regions of space. Now, measuring "in a region" implies a position measurement, and this fact has been underestimated until now. Let us consider a typical EPR experiment: Two signals (that I shall call particles in the following) arrive at two separated regions, and if $A_{j}$ and $B_{j}$ are two observables with range $\{0,1\}$ associated with particle $j(j=1$ or 2 ), the experiment consists of measuring the one-particle observables $A_{j} R_{j}$ and $B_{j} R_{j}$, and the two-particle ones obtained as a product of these. Here $R_{j}$ is the observable taking value $1(0)$ if particle $j$ is (is not) inside the corresponding region, and we define the product of observables to be another observable taking value 1 if, and only if, both terms in the product take value 1 . With these definitions, the Clauser and Horne [9] inequality (2.5) should be written

$$
\begin{aligned}
\left\langle B_{1} R_{1}\right\rangle+\left\langle A_{2} R_{2}\right\rangle \geq & \left\langle A_{1} R_{1} A_{2} R_{2}\right\rangle+\left\langle B_{1} R_{1} A_{2} R_{2}\right\rangle \\
& +\left\langle B_{1} R_{1} B_{2} R_{2}\right\rangle-\left\langle A_{1} R_{1} B_{2} R_{2}\right\rangle,
\end{aligned}
$$

where \langle\rangle means average value. In order to show a conflict between quantum mechanics and the Bell inequality (3.1), we should find a wave vector $|\psi\rangle$ and projection operators $A_{j}, B_{j}, R_{j}$, violating the inequality with

$$
\begin{aligned}
& \left\langle A_{1} R_{1}\right\rangle=\left\langle\psi\left|A_{1} R_{1}\right| \psi\right\rangle, \\
& \left\langle A_{1} R_{1} A_{2} R_{2}\right\rangle=\left\langle\psi\left|A_{1} R_{1} A_{2} R_{2}\right| \psi\right\rangle \text {, etc. }
\end{aligned}
$$

The explicit use of the position observables $R_{j}$ may con- tribute to clarify the real meaning of the Bell inequalities, a subject that has been misunderstood many times. For instance, an inequality like

$$
\begin{aligned}
\left\langle B_{1}\right\rangle+\left\langle A_{2}\right\rangle \geq & \left\langle A_{1} A_{2}\right\rangle+\left\langle B_{1} A_{2}\right\rangle \\
& +\left\langle B_{1} B_{2}\right\rangle-\left\langle A_{1} B_{2}\right\rangle
\end{aligned}
$$

might be interpreted without any reference to locality if it is not explicitly stated that the measurement of $A_{1}$ and $B_{1}$ should be made in a region and that of $A_{2}$ and $B_{2}$ in another separated region, that is if (5.3) is not understood as a simplified form of writing (5.1). Indeed, from time to time, it has been claimed that the Bell inequalities are unrelated to locality, but derive from the mere existence of a joint probability distribution for four observables, not all compatible. This error rests upon the confusion between Bell inequalities like (5.1), valid for all local theories, and less general inequalities, with the same form of (5.3), valid only for noncontextual hidden-variable theories. A lot of misunderstandings should be avoided if Bell's name were used only for the first class of inequalities.

Also, the need to antisymmetrize (symmetrize) the wave function of many-fermion (many-boson) systems has been considered a source of nonlocality. That this is not true was pointed out many years ago by Ghirardi et al. [20] and it can be easily understood with the aid of the observables $R_{j}$. For instance, it is obvious that if we have two spin- $\frac{1}{2}$ particles localized in two separated regions, the first particle on the left with spin up, and the second one on the right with spin down, no problem with locality exists and (5.1) is indeed fulfilled. Now, if the two particles are identical and we use an antisymmetrized wave function (and symmetrized operators), again (5.1) is fulfilled because the value of every term in that inequality remains unchanged by the antisymmetrization procedure. That is, the particle on the left (right) always has spin up (down), although now the labels first or second are not attached to one position (and spin projection) each. However, without the introduction of the operators $R_{j}$ we might be mislead to the conclusion that a Bell inequality could be violated by the antisymmetrized (entangled) wave function.

In the proofs of Bell's theorem it is common to exhibit a conflict between quantum mechanics and inequalities like (5.3). However, the proof of a contradiction with LHV theories is only valid if (5.3) is understood in the sense of (5.1), as I have shown. However, this fact is not clear in most of the published proofs of Bell's theorem, which are therefore incomplete. A similar criticism applies to recent proofs of the theorem without using inequalities [21], where only the spin part of some three- or four-particle system is considered, without any analysis of the directional correlation of these particles.

In order to test quantum mechanics against LHV theories it is necessary to find an experiment such that the quantum-mechanical predictions violate inequality (5.1). A requirement is that two particles must be produced with a strong directional correlation, besides good correlation of spin (or other appropriate quantity). In order to have a good angular correlation we might use two-body processes and several experiments of this kind 
have been discussed in the literature [2-4], such as proton-proton scattering, positronium annihilation, dissociation of a diatomic molecule [22], or high-energy tests. However, all these proposals present great difficulties, as shown by the fact that 26 years after the Bell work no experiment has been performed or is planned for the near future, which is a reliable test of LHV theories against quantum mechanics. Therefore, it is interesting to explore other possibilities. In the domain of optics it does not seem possible to use two-body processes, but a good directional correlation of photon pairs might be obtained by one of the following two methods.

1. It would be possible to use a three-body decay if the recoil atom were detected. The proposal is to improve the performed photon polarization correlation experiments (by Aspect and others) by measuring the polarization correlation on the restricted ensemble of photon pairs produced in decays that leave the atom with a welldefined linear momentum. (This ensemble is well defined operationally, at a difference with others mentioned in the literature, like the "ensemble of pairs such that both photons pass through the apertures," commented on in Sec. IV.) These photon pairs will possess a good angular correlation if the atoms before emission also had a good definition of momentum.

2. It seems possible to produce entangled photon pairs with well-correlated wave vectors by parametric down conversion, and some experiments have been performed by measuring either phase correlation [23] or polarization correlation [24]. These experiments might be improved in order to test genuine Bell inequalities (i.e., not involving supplementary assumptions).

We comment on the first possibility because it throws light on the relevance of the LHV models presented in Sec. II for the atomic cascade experiments. In these experiments we may consider the following set of five compatible observables: the two momenta of the photons, their two polarizations and the momentum of the recoil atom. In the performed experiments, only the first four observables have been measured (photon momenta through the measurement of the angle of emission) so that the quantum predictions Eqs. (2.1) are actually averages over the final momenta of the recoil atoms. The question arises whether LHV models exist predicting the correlation of the five observables in agreement with quantum mechanics. Equations (2.8), (2.9), and (2.13) of the models exhibited in Sec. II would then be averages over the hidden variables of the recoil atom, of the equations of those more complete models. The answer to the question seems to be negative, as shown by the following argument.

We may consider experiments where we select (by a time-of-flight method, for instance) an ensemble of atoms having all the same initial velocity. (In the experiments by Aspect and co-workers [6-8], as well as in most other experiments, the atoms come from a thermal source and they have a more or less Maxwellian distribution of velocities.) Then, we select (by recording only coincidence counts of an atom with one or two photons) those emitted photon pairs, where the recoil atom has a well-defined momentum. In this way, we have an operationally defined ensemble of photon pairs with known total momentum. For this ensemble, assumptions (i) to (iv) of Sec. IV presumably hold [if (A) also holds, which we shall discuss in Sec. VI]. Choosing the total momentum of the photon pair in such a way that the photons travel in directions forming an angle close to $\pi$ [so that the function $F$ given in (2.4) is close to one], then the quantum predictions for this ensemble probably contradict a Bell inequality. This would imply that LHV models for the experiment are not possible (except relying on the low efficiency of detectors) so that we have here a possibility of blocking the "poor correlation" loophole. A detailed study of this experiment is in progress.

\section{LOOPHOLES DUE TO IMPERFECT APPARATUSES}

Let us now assume that an experiment is possible combining good directional correlation with good polarization correlation (or correlation of spin or other appropriate quantity) and such that QM predictions contradict LHV for this experiment. The counterexample of Sec. III proves that in order to have a real conflict between LHV theories and the results of the experiment, we still need to use apparatuses close enough to the ideal. (We should mention that there are two additional loopholes related to the static nature of the whole device. The first one is due to the possibility of communication between the two sides of the measuring apparatus; the third experiment by Aspect, Dalibard, and Roger [8] was devised with the purpose of blocking this loophole. The second one is due to possible memory effects in the polarizers and/or detectors [25,26].)

Imperfections can be associated with both the source and the measuring devices. There are at least two problems with the source, which we shall not analyze here, but we should mention for the sake of completeness. For clarity we shall consider atomic cascade experiments such as those analyzed in Sec. II, but the argument is general. In the first place, if the decay rate in the source is too high, then different events - each consisting of an atomic decay followed by a measurement-cannot be discriminated. The problem of the so-called "accidental coincidences" appears (e.g., detection of two photons, each coming from a different atom). It is possible to estimate the amount of noise produced by these coincidences and subtract it, but the procedure is subject to criticisms [27]. The correct solution is to use a low enough decay rate, as has been done in several experiments [28]. The second problem appears if the source is too big and/or the density of atoms in the source is too high. In this case some of the photons may suffer rescattering giving rise to a possible bias in the measured correlation [29].

Difficulties with the measuring devices come either from the selector (polarizer for optical photons, SternGerlach for spin- $\frac{1}{2}$ atoms, etc.) or from the detector, and it seems that the combination selector-detector is never good enough. In fact, in the case of photons, where transmission through a polarizer corresponds to wave behavior and detection to particle behavior, it is not strange that we have better polarizers as the wavelength increases 
(e.g., for optical photons) and better detectors as the wavelength decreases (e.g., for gamma rays). In the case of atoms, where the particle behavior is clearly dominant, the efficiency of the Stern-Gerlach is not enough to make a reliable experiment (see the end of Shimony's repiy in Ref. [22]). We have here a hint that the problem of imperfections in the measuring devices may have a fundamental character rather than being just a practical difficulty to be solved with future technology. We shall comment on this in the next section.

From now on we shall discuss only the low-efficiency loophole, which is most relevant for the atomic cascade experiments. As is well known, performed experiments have used detectors with efficiencies in the range $10-30 \%$, while the violation of the Bell inequality (2.5) cannot be produced with efficiencies smaller than $82 \%$ [12]. It has been argued that it should be unlikely, almost conspiratory, if the correlations measured with highefficiency detectors were different from the correlations actually measured with low-efficiency ones [30]. It is not so. Indeed, the change of correlation with efficiency is quite natural, almost compulsory, in any hidden-variable theory, as can be seen by the following argument.

The core of a hidden-variable theory is the assumption that systems that are identical according to quantum theory are actually not identical. For instance, two atoms in the same excited state decay at different times. According to orthodox quantum mechanics this is a manifestation of the "essential acausality of nature." In sharp contrast, any reasonable hidden-variable theory should try to explain atomic behavior causally by assuming that those atoms are not identical, but our information about them-derived from what we can control in their preparation - is identical. In other words, a pure quantum state will correspond to a mixture in the hiddenvariable theory. This is just the meaning of the function $\rho(\lambda)$ in the Bell definition of LHV theory, Eqs. (2.6) and (2.7). Obviously, photons having different $\lambda$ will behave differently, and this includes the whole process of crossing the polarizer and being detected. If one has in mind a mechanistic picture of the photon, derived from a naive interpretation of the quantum formalism as discussed in Sec. IV, then one is lead to assume that, although the probability of crossing the polarizer may depend on $\lambda$, the detection probability, once the photon has passed, should not. Hence the assumption (i) of Clauser et al. [10], discussed in Sec. IV, follows. However, as we have argued in that section, any LHV theory able to explain the results of the performed experiments does not allow writing the full probability as a product of "probability of passing" the polarizer times "probability of being detected" conditional to passage. That is, in any LHV theory compatible with performed experiments, we must consider that the detection probability of a signal (photon) arriving at a detector does depend on $\lambda$. This assumption leads quite naturally to the prediction that the measured correlation will decrease with detector efficiency, as we show in the following.

For the sake of clarity I consider a simple hiddenvariable model of a macroscopic detector as consisting of a sequence of $N$ atoms with which the incoming signal in- teracts one after the other. Let us assume that the activation of any atom produces a count in the detector. If $\sigma(\lambda)$ is the (very small) probability that a signal with hidden variable $\lambda$ activates an atom (we assume for simplicity that it is the same for all atoms), then the probability that a signal entering, maybe partially, the detector activates the $n$th atom will be $\sigma(1-\sigma)^{n-1}$ and the probability of a count

$$
\zeta(\lambda)=\sum_{n} \sigma(1-\sigma)^{n-1}=1-(1-\sigma)^{N} \approx 1-\exp [-\sigma(\lambda) N] .
$$

We see that for low efficiencies $(\sigma N \ll 1)$ all single (coincidence) probabilities scale linearly (quadratically) with the average efficiency $\eta=\langle\zeta(\lambda)\rangle_{\lambda}$, but it is not so for higher ones. This means that although correlations remain the same in the low efficiency (linear) region, they may drastically change when the efficiency approaches unity. In order to illustrate this fact more clearly we present a LHV model for the polarization correlation of optical-photon pairs, involving the detection Eq. (6.1).

We shall try to reproduce the quantum predictions for a hypothetical experiment having good directional correlation, such as the experiment involving the detection of the recoil atom, as discussed in Sec. V. These predictions are

$$
\begin{aligned}
& p_{1}(a)=p_{2}(b)=\frac{1}{2} \eta, \\
& p(a, b)=\frac{1}{4} \eta^{2}[1+F \cos (2 a-2 b)],
\end{aligned}
$$

where for simplicity we consider single-channel polarizers. In order to simplify the model, emphasizing only the role of the detector efficiency, we have put equal to unity a number of factors involved in real experiments [compare Eq. (6.2) with (2.1)]. We construct a LHV model where $\lambda$ consists of two hidden variables $\left\{\mu_{1}, \mu_{2}\right\}$, both with range $[0, \pi]$, and we use the density function

$$
\rho(\lambda)=\pi^{-2}\left[1+\cos \left(2 \mu_{1}-2 \mu_{2}\right)\right] .
$$

We begin assuming that our detectors consist of a single atom each, and the functions $P_{1}(\lambda, a)$ and $P_{2}(\lambda, b)$ are

$$
\begin{aligned}
P_{j}(\lambda, a) & =\sigma(\lambda, a)=\exp \{-\beta x], \\
x & =\min \left\{\left|\mu_{j}-a\right|,\left|\mu_{j}-a-\pi\right|,\left|\mu_{j}-a+\pi\right|\right\} .
\end{aligned}
$$

This gives

$$
\begin{gathered}
p_{1}(a)=p_{2}(b)=(\pi \beta)^{-1}[1-\exp (-\pi \beta / 2)], \\
p(a, b)=p_{1}^{2}\left\{1+\left[\beta^{2} \operatorname{coth}(\pi \beta / 2) /\left(\beta^{2}+4\right)\right]^{2}\right. \\
\times \cos (2 a-2 b)\},
\end{gathered}
$$

and perfect agreement with QM is obtained by choosing

$$
\begin{aligned}
& \eta=(\pi \beta / 2)^{-1}[1-\exp (-\pi \beta / 2)], \\
& F=\left[\beta^{2} \operatorname{coth}(\pi \beta / 2) /\left(\beta^{2}+4\right)\right]^{2} .
\end{aligned}
$$

Now we consider detectors containing a large number $N$ of active atoms each. Then, combining (6.4) with (6.1), we get 


$$
\begin{aligned}
P_{j}(\lambda, a) & =1-\exp [-N \exp (-\beta x)] \\
& \cong 1 \quad \text { for } x<\gamma, \quad 0 \text { for } x>\gamma,
\end{aligned}
$$

where

$$
\gamma=\beta^{-1} \ln N .
$$

[We note that (6.7) is similar to (2.9).] Hence we get

$$
\begin{aligned}
& p_{1}(a)=p_{2}(b)=2 \gamma / \pi, \\
& p(a, b)=p_{1}^{2}\left\{1+[\sin (2 \gamma) / 2 \gamma]^{2} \cos (2 a-2 b)\right\} .
\end{aligned}
$$

We see that perfect agreement with $\mathrm{QM}$ is no longer possible. In fact, if we choose

$$
\eta=4 \gamma / \pi,
$$

in order to have agreement for $p_{1}$, then we get for $N \gg 1$, $p(a, b)=\frac{1}{4} \eta^{2}\left\{1+\left[1-\pi^{2} \eta^{2} / 12+O\left(\eta^{4}\right)\right] \cos (2 a-2 b)\right\}$,

which clearly exhibits a decrease in correlation with increasing detector efficiency.

It is remarkable that this LHV model, which is proposed just for illustrative purposes, allows fitting all the relevant quantities in good agreement with real experiments. For instance, if we take $F \cong 0.99$, which is a typical value [see Eq. (2.4)], we get from (6.6) the value $\beta \cong 30$. Then, for the efficiencies used in performed experiments $(\eta \approx 0.3)$, Eqs. (6.10) and (6.8) give $N \cong 10^{3}$. Finally, we obtain from (6.11) departures from QM of the order of $1-2 \%$, which are within the statistical errors of the experiments. Very likely, any reasonable hiddenvariable model of the detection process has a similar behavior (it is true, for instance, for the more sophisticated model described elsewhere [31]).

We conclude that although a linear extrapolation (assumption A of Sec. IV) of the measured polarization correlations to higher efficiencies would violate the Bell inequality (2.5), this fact does not imply that a violation will be produced if the experiments are actually performed with more efficient detectors.

\section{DISCUSSION}

We have proved that no experimental test of a Bell inequality has shown a true refutation of LHV theories or local realism. Furthermore, no experiment, performed or planned, is able to show a contradiction between quantum mechanics and LHV theories. Only highly idealized (gedanken) experiments have shown that conflict. Consequently I can safely claim that the problem of whether a local realistic picture of the physical world is possible remains open. The problem is important and worth studying. Therefore efforts, both theoretical and experimental, should be addressed to it.

We seek the following possibilities for the future.

(i) No experiment is ever found suitable to show a contradiction between quantum mechanics and local realism.

(ii) An experiment is eventually performed in which the predictions of quantum mechanics show an actual con- tradiction with local realism, and the results contradict quantum mechanics; and

(iii) The above experiment agrees with quantum mechanics and refutes all LHV theories.

I shall comment only on the first possibility.

The conflict between quantum mechanics and local realism is usually shown by exhibiting a state vector and several projection operators in a suitable Hilbert space, such that Eqs. (5.2) lead to a violation of the Bell inequality (5.1). It is obvious that with present technologywith any technology, in fact-it is possible neither to prepare the states corresponding to all quantum state vectors nor to measure the observables corresponding to all quantum projection operators. However, it is usually assumed that no difficulty of principle exists. In other words, it is taken as one of the postulates of quantum mechanics that all vectors in the Hilbert space represent physical states and all self-adjoint operators represent observables, except for the superselection rules. If this postulate is accepted, then certainly quantum mechanics contradicts local realism.

My conjecture is that the above postulate could be replaced by another one, whose precise formulation is not yet known, stating that only some subset of quantum states and observables are physical and that these do not contradict local realism. In other words, some modification could be introduced in the formalism of quantum mechanics making it compatible with local realism, but preserving the existing perfect agreement with all experiments. The fact that no experiment able to discriminate between quantum mechanics and the whole family of LHV theories has been performed until now shows that the conjecture has not yet been refuted.

In order to explain the conjecture more clearly, consider an example. If we want to perform an EPR-type experiment, we must measure observables like $A_{j} R_{j}$ discussed in Sec. V. Let us assume that we use photons and and that $A_{j}$ means linear polarization. Now, polarization is a wave property, while position (associated to $R_{j}$ ) is a particle property. It might be that the product $A_{j} R_{j}$ cannot be measured so accurately as to test locality. For example, the region associated to $R_{j}$ may be quite small for gamma rays, but not so small for optical photons (e.g., if the aperture of the lens system is too small, diffraction phenomena become important). In contrast, polarization of optical photons can be accurately measured, while no accurate polarizer exists for gamma rays.

Using the quantum formalism, the example can be put this way. QM assumes that it is possible, in principle, to prepare a single photon signal either with polarization in the vertical plane, represented by the state vector $|V\rangle$, or in the horizontal plane with state vector $|H\rangle$, or in other polarization states, with state vectors that are linear combinations of the previous two. Similarly, it is assumed that there exist in principle measuring devices corresponding to the projection operator $V(H)$ having eigenvalue $1(0)$ for the state vector $|V\rangle$ and 0 (1) for the state vector $|H\rangle$. In practice, however, such devices will consist of a polarizer and a detector, and they give quite different results. In fact, when a photon in state $|\boldsymbol{V}\rangle$ is measured with the device corresponding to $V$ we get the 
number 1 (i.e., a count in the detector) with probability $\eta \epsilon_{+}$(typically $\eta \approx 0.2, \epsilon_{+} \approx 0.98$ ) and the number 0 (i.e., no counts) with probability $1-\eta \epsilon_{+}$. On the other hand, if the incoming photon is in the state $|H\rangle$, we get 1 with probability $\eta \epsilon_{-}$(typically $\epsilon_{-} \approx 0.2$ ) and 0 with probability $1-\eta \epsilon_{-}$. Then we may say that our polarizer-detector device does not correspond to the operator $V$, but to some projection operator with the same expectations as the operator $\eta\left(\epsilon_{+} V+\epsilon_{-} H\right)$. My conjecture is that the range of possible values of $\eta, \epsilon_{+}$, and $\epsilon_{-}$for real devices may not include those actually needed to violate a genuine Bell inequality. It must be taken into account that maybe the parameters $\eta\left(\epsilon_{+}\right.$and $\left.\epsilon_{-}\right)$cannot be attributed to the detector (polarizer) alone, so that $\epsilon_{+}$and/or $\epsilon_{-}$may change when the efficiency $\eta$ is increased.

Of course, this has been just an example. What I conjecture is, first, that nature can be interpreted within local realism. I am firmly convinced that this part of the conjecture is true. The second part is that this fact does not necessarily imply a failure of quantum mechanics, provided we restrict the domain of application of the theory by limiting the set of operators associated to observables and the set of vectors corresponding to physical states. I am less sure about this part. A study of new experiments, as those considered at the end of Sec. V, may allow disproving the conjecture. In contrast, if these experiments prove to be unreliable, as has happened with all experiments performed or planned in the past, the conjecture will be reinforced.

\section{ACKNOWLEDGMENT}

I acknowledge financial support of DGICYT Project No. PS-90-0098 (Spain).
[1] J. S. Bell, Physics (N.Y.) 1, 195 (1964).

[2] J. F. Clauser and A. Shimony, Rep. Prog. Phys. 41, 1881 (1978).

[3] Quantum Mechanics versus Local Realism: The Einstein, Podolsky and Rosen Paradox, edited by F. Selleri (Plenum, New York, 1988).

[4] D. Home and F. Selleri, Riv. Nuovo Cimento 14 (9), 1 (1991).

[5] E. Santos, Phys. Rev. Lett. 66, 1388 (1991).

[6] A. Aspect, P. Grangier, and G. Roger, Phys. Rev. Lett. 47, 460 (1981).

[7] A. Aspect, P. Grangier, and G. Roger, Phys. Rev. Lett. 49, 91 (1982)

[8] A. Aspect, J. Dalibard, and G. Roger, Phys. Rev. Lett. 49, 1804 (1982).

[9] J. F. Clauser and M. A. Horne, Phys. Rev. D 10, 526 (1974).

[10] J. F. Clauser, M. A. Horne, A. Shimony, and R. A. Holt, Phys. Rev. Lett. 23, 880 (1969).

[11] E. Santos, in Proceedings of the Conference on "Bells's Theorem and the Foundations of Modern Physics", edited by A. van der Merwe (Plenum, New York, in press).

[12] A. Garg and D. N. Mermin, Phys. Rev. D 35, 3831 (1987).

[13] J. von Neumann, Mathematical Foundations of Quantum Mechanics (Princeton University Press, Princeton, NJ, 1955) (German original, 1932).

[14] J. S. Bell, Rev. Mod. Phys. 38, 447 (1966).

[15] A. Garuccio and V. Rapisarda, Nuovo Cimento A 65, 269 (1981).

[16] P. Grangier, M. J. Potasek, and B. Yurke, Phys. Rev. A 38, 3132 (1988).
[17] E. Santos, Phys. Rev. Lett. 68, 894 (1992); S. M. Tan, D. S. Walls, and M. J. Collett, ibid. 68, 895 (1992).

[18] E. Santos, Phys. Rev. Lett. 68, 2702 (1992).

[19] T. W. Marshall and E. Santos, Found. Phys. 18, 185 (1988); Phys. Rev. A 39, 6271 (1989).

[20] G. C. Ghirardi, A. Rimini, T. Weber, and C. Omero, Nuovo Cimento B 39, 130 (1977).

[21] D. M. Greenberger, M. A. Horne, A. Shimony, and A. Zeilinger, Am. J. Phys. 58, 1131 (1990).

[22] T. K. Lo and A. Shimony, Phys. Rev. A 23, 3003 (1981); E. Santos, ibid. 30, 2128 (1984); A. Shimony, ibid. 30, 2130 (1984).

[23] J. G. Rarity and P. R. Tapster, Phys. Rev. Lett. 64, 2495 (1990).

[24] Z. Y. Ou and L. Mandel, Phys. Rev. Lett. 61, 50 (1988); Y. H. Shih and C. O. Alley, ibid. 61, 2921 (1988); L. Hardy, Phys. Lett. A 161, 326 (1992).

[25] E. Santos, Phys. Lett. A 101, 379 (1984).

[26] S. Pascazio, Phys. Lett. A 118, 47 (1986).

[27] E. Santos, in Quantum Uncertainties, edited by W. H. Honig (Plenum, New York, 1987), pp. 313-330.

[28] A. Aspect and P. Grangier, Lett. Nuovo Cimento 45, 435 (1985). Other experiments where this problem did not appear are those of Clauser and Freedman and of Holt and Pipkin, commented on in Refs. [2-4].

[29] F. Selleri, Lett. Nuovo Cimento 39, 252 (1984).

[30] See, for instance, D. Mermin, in New Techniques and Ideas in Quantum Measurement Theory, edited by $\mathrm{D}$. Greenberger (New York Academy of Sciences, New York, 1986), p. 422.

[31] E. Santos, Phys. Lett. A 139, 431 (1989). 